lowable bandwidth compared with $\mathrm{BPC}$, resulting in a factor of two increase in the range resolution of the radar. The MSK waveform also has been demonstrated to have an ambiguity sidelobe structure very similar to BPC, where the sidelobe levels can be decreased by increasing the length of the $\mathrm{m}$-sequence used in its generation.

This ability to set the peak sidelobe level is advantageous as it allows the sys- tem to be configured to a variety of targets, including those with a larger dynamic range. Other conventionally used waveforms that possess an even greater spectral efficiency than the MSK waveform, such as linear frequency modulation (LFM) and Costas frequency hopping, have a fixed peak sidelobe level that is therefore not configurable, and can be exceeded by high contrast targets. Furthermore, in the case of a mul- tistatic experiment observing a target in motion, self-interference from the transmitter to the receiver is mitigated by the MSK waveform. Waveforms that have delay Doppler coupling, such as LFM, provide no such protection.

This work was done by Kevin J. Quirk and Meera Srinivasan of Caltech for NASA's Jet Propulsion Laboratory. For more information, contact iaoffice@jpl.nasa.gov. NPO48367

\title{
(9) Telescope Alignment From Sparsely Sampled Wavefront Measurements Over Pupil Subapertures
}

\section{NASA's Jet Propulsion Laboratory, Pasadena, California}

Alignment of two-element telescopes is a classic problem. During recent integration and test of the Space Interferometry Mission's (SIM's) Astrometric Beam Combiner (ABC), the innovators were faced with aligning two such telescope subsystems in the presence of a further complication: only two small subapertures in each telescope's pupil were accessible for measuring the wavefront with a Fizeau interferometer.

This meant that the familiar aberrations that might be interpreted to infer system misalignments could be viewed only over small sub-regions of the pupil, making them hard to recognize. Further, there was no contiguous surface of the pupil connecting these two subapertures, so relative phase piston information was lost; the underlying full-aperture aberrations therefore had an additional degree of ambiguity.
The solution presented here is to recognize that, in the absence of phase piston, the Zygo measurements primarily provide phase tilt in the subaperture windows of interest. Because these windows are small and situated far from the center of the (inaccessible) unobscured full aperture, any aberrations that are higher-order than tilt will be extremely high-order on the full aperture, and so not necessary or helpful to the alignment. Knowledge of the telescope's optical prescription allows straightforward evaluation of sensitivities (subap mode strength per unit full-aperture aberration), and these can be used in a predictive matrix approach to move with assurance to an aligned state.

The technique is novel in every operational way compared to the standard approach of alignment based on full-aper- ture aberrations or searching for best rms wavefront. This approach is closely grounded in the observable quantities most appropriate to the problem. It is also more intuitive than inverting full phase maps (or subaperture Zernike spectra) with a ray-tracing program, which must certainly work in principle, but in practice met with limited success. Even if such classical alignment techniques became practical, the techniques reported here form a reassuringly transparent and intuitive check on the course of the alignment with very little computational effort.

This work was done by Eric. E. Bloemhof, Xin An, Gary M. Kuan, Douglas M. Moore, Joseph F. O'Shay, Hong Tang, and Norman A. Page of Caltech for NASA's Jet Propulsion Laboratory. For more information, contact iaoffice@jpl.nasa.gov. NPO47814

\section{Method to Remove Particulate Matter From Dusty Gases at Low Pressures}

\section{This method could be of use to the semiconductor industry to remove particles during low- pressure plasma processing.}

\section{John F. Kennedy Space Center, Florida}

Future human exploration of Mars will rely on local Martian resources to reduce the mass, cost, and risk of space exploration launched from Earth. NASA's In Situ Resource Utilization (ISRU) Project seeks to produce mission consumables from local Martian resources, such as atmospheric gas. The Martian atmosphere, however, contains dust particles in the 2-to-10-micrometer range.
These dust particles must be removed before the Martian atmospheric gas can be processed. The low pressure of the Martian atmosphere, at 5 to 10 mbars, prevents the development of large voltages required for a standard electrostatic precipitator. If the voltage is increased too much, the corona transitions into a glow/streamer discharge unsuitable for the operation of a precipitator. If the voltage is not large enough, the dust particles are not sufficiently charged and the field is not strong enough to drive the particles to the collector.

A method using electrostatic fields has been developed to collect dust from gaseous environments at low pressures, specifically carbon dioxide at pressures around 5 to 10 mbars. This method, 